\title{
TURISMO RURAl COMUNITARIO EN MÉXICO: APUNTES PARA FUTURAS INVESTIGACIONES
}

\author{
Maxime Kieffer \\ mkieffer@enesmorelia.unam.mx \\ Escuela Nacional de Estudios Superiores, Morelia, \\ Universidad Nacional Autónoma de México
}

El objetivo del ensayo fue analizar la relación entre el turismo rural comunitario (TRC) y el desarrollo comunitario en México, sus retos y alcances, para entender mejor el fenómeno y sus implicaciones para las comunidades rurales a nivel social, cultural, económico y ambiental. La reflexión, enriquecida por alrededor de doce años de trabajo de campo sobre el tema, investigaciones formales, pláticas y debates en espacios académicos y con actores locales, sistematizó los puntos de encuentro de las reflexiones actuales y evidenció algunos temas pendientes de investigación sobre el turismo rural comunitario.

Palabras clave: México, comunidades rurales, desarrollo y conservación, investigación interdisciplinaria, turismo rural comunitario.

\section{Community based rural tourism in Mexico: NOTES FOR FUTURE RESEARCH}

This paper presents an interdisciplinary reflection on the relationship between Community-based Rural Tourism (CBRT) and community development in Mexico, highlighting its successes and main issues, a better understand this issue and its implications for rural communities on the social, cultural, economic, and environmental levels. This reflection has been enriched by approximately twelve years of field work, formal research, discussions and debates in academic spaces with local stakeholders. It has also systematized the agreement points on current issues as well as exposing some pending research tasks about CBRT.

Keywords: Mexico, interdisciplinary research, Community-based Rural Tourism, conservation and development, rural communities.

Fecha de recepción: 7 de febrero 2019. Fecha de aceptación: 25 de junio de 2019

CÓMO CITAR: Kieffer, M. (2019). Turismo rural comunitario en México: apuntes para futuras investigaciones. Dimensiones Turísticas, 3(5), 43-63. https://doi.org/10.47557/XSNY8857 


\section{Introducción}

A partir de la década de los noventa, varios factores combinados cambiaron el panorama tanto del medio rural como del turismo: la crisis del medio rural inducida por las políticas agrarias y los tratados de libre comercio; la degradación de la situación ambiental y el tema de la conservación de los recursos naturales; las políticas internacionales de impulso al desarrollo sustentable; así como la diversificación de actividades turísticas. Como consecuencia, los países de América Latina, y en particular México, apostaron a la introducción del turismo en el medio rural como política de desarrollo y de lucha contra la pobreza y como política ambiental de conservación de los recursos. Aunado a esto, la creciente presión por minimizar los impactos negativos del turismo convencional y por diversificar la oferta turística en el país, basada en los principios del turismo sustentable, hizo emerger nuevas formas de turismo, comúnmente llamadas turismo alternativo (Ávila Romero, 2015; Velarde Valdez, Maldonado Alcudia y Gómez Nieves, 2015). Aunque la denominación “alternativo" pueda remitir a varias situaciones, desde una actividad turística diferente hasta un modelo de gestión colectiva del turismo, la particularidad de la organización territorial colectiva en México, a través de los ejidos y de sus asambleas para las tomas de decisiones locales, dio paso a un tipo de turismo particular denominado turismo rural comunitario (TRC). Esta denominación de TRC también aparece en otros países latinoamericanos, tales como Perú, Ecuador, Bolivia, entre otros, en donde prevalece igualmente una gestión colectiva del territorio. Cañada (2015, p. 160) define el TRC como:

Un tipo de turismo desarrollado en zonas rurales en el que la población local, a través de distintas estructuras organizativas de carácter colectivo, ejerce un papel protagónico en su desarrollo, gestión y control, ofreciendo actividades respetuosas con el medio natural, cultural y social, y con los valores de una comunidad, que permite disfrutar de un positivo intercambio de experiencias entre residentes y visitantes, donde la relación entre el turista y la comunidad es justa y los beneficios de la actividad son repartidos de forma equitativa.

El enraizamiento de los discursos internacionales acríticos sobre la sinergia entre el turismo, el desarrollo en el medio rural y la conservación del medioambiente tuvo como consecuencia que el desarrollo del turismo se volviera central en las políticas públicas mexicanas. Así, varias instituciones gubernamentales tales como la Comisión Nacional para el Desarrollo de los Pueblos Indígenas (CDI), la Secretaría de Medio Ambiente y Recursos Naturales (Semarnat), la Secretaría de Desarrollo Social (Sedesol), la Secretaría de Turismo (Sectur), para citar las más importantes, a través de distintos programas a nivel federal y estatal, así como organismos internacionales como la Organización Mundial del Turismo (омт) y la Organización de las Naciones Unidas (ONU), impulsaron, con resultados fluctuantes, el desarrollo del turismo en el medio rural bajo la forma de TRC. El inicio de estas políticas públicas para desarrollar el turismo en el medio rural remonta a la mitad de los noventa y los primeros financiamientos de centros ecoturísticos; por ejemplo, Ara 
Macao Las Guacamayas y Las Nubes en Chiapas; Angahuan en Michoacán; Los Pueblos Mancomunados en Oaxaca; y Ek Balam en Yucatán.

El auge del financiamiento de estos centros ecoturísticos se sitúa entre los años 2005 y 2015, con una multiplicación de proyectos turísticos de base comunitaria, financiados por inversiones públicas. Hoy en día, aunque la situación sea muy cambiante, estudios han contabilizado más de 2500 empresas existentes en este sector (López Pardo y Palomino Villavicencio, 2014). Así, el sector del turismo en el medio rural, y en particular el TRC, ha sido un sector de mucho crecimiento, con una evolución de las prácticas turísticas muy rápida; es decir, un sector dinámico y fluctuante, con iniciativas nuevas naciendo de manera constante, y otras abandonando en camino.

La emergencia de estas nuevas formas de turismo ha sido acompañada de una evolución de las temáticas de investigación en turismo, basada en conceptos y marcos teóricos nuevos para los académicos del turismo (Kieffer y Jouault, 2017). Al implantarse en contextos rurales particulares, con formas de organización social específicas, condiciones ambientales y culturales distintas, la relación que el turismo mantiene con las comunidades en esos espacios ha sido de gran interés para la comunidad científica. La relación entre el turismo y las sociedades rurales en las esferas ambientales, sociales, políticas, económicas y culturales, es cada vez más presente en los espacios académicos, no solamente de turismo, sino de varias disciplinas de las humanidades y ciencias sociales. Diversas disciplinas se han acercado al fenómeno del TRC, cada una contribuye a observar los procesos que involucra, a través del prisma de los enfoques teóricos y metodológicos de cada disciplina, e incluso a partir de enfoques multidisciplinarios.

En este contexto, es de particular interés notar que la investigación en turismo en México está vinculada con la problemática del desarrollo, específicamente del desarrollo en el medio rural, y con los efectos del turismo en las comunidades rurales sobre cuestiones tan diversas como su relación con la lucha contra la pobreza (Dehoorne, Tatar y Theng 2013; Girard y Schéou, 2012; Manyara y Jones, 2007; Organización Mundial del Turismo, 2014), sus lazos con la conservación ambiental y las políticas públicas vinculadas (García-FrapoIli, Ramos-Fernández, Galicia y Serrano, 2009; Obombo Magio y Velarde Valadez, 2018; Vargas del Río y Brenner, 2013), su relación con la cultura (Santana, 2009), las formas de organización social para el TRC (Kieffer, 2018), y muchos subtemas inducidos por el impulso de estas formas de turismo en el medio rural. Tan es así que numerosos congresos y seminarios académicos y cumbres internacionales de organismos no gubernamentales tienen desde aproximadamente el 2010 por tema central el turismo en el medio rural y su relación con el desarrollo y la conservación. El TRC plantea el tema de la pobreza y del desarrollo de los territorios y sociedades rurales (Cañada y Gascón, 2007); propone ofrecer a las comunidades rurales la posibilidad de mejorar el control que ejercen sobre su propia política de desarrollo local, vinculándose en los ámbitos sociales, económicos y ambientales; pretende reafirmar las identidades locales, favorecer la organización comunitaria y promover la autogestión y la gobernanza local. Ahora que existe la posibilidad de tomar un poco de distancia para contemplar el camino transcurrido, y realizar balances de las iniciativas existentes, 
surge la necesidad de comparar las expectativas y promesas teóricas con lo que ocurre en el terreno. Esto permitiría, por un lado, diagnosticar logros y problemas, mejorar el diseño de nuevos proyectos y cuestionar el abordaje de las cuestiones relacionadas con el TRC y el desarrollo comunitario por los diversos actores implicados, desde las comunidades hasta los académicos, incluyendo promotores, asociaciones civiles y agentes gubernamentales.

\section{El turismo rural comunitario en debate}

En México, los primeros estudios académicos en el ámbito del turismo alternativo empiezan a mediados de los años noventa y, por el interés que suscita este sector de actividad y su rápido crecimiento a nivel internacional y nacional, los estudios se multiplicaron. Si bien cada vez hay más estudios sobre el tema, las prácticas turísticas evolucionan y cambian tan rápidamente la realidad estudiada, que es importante mantener una atención constante sobre el fenómeno. Hasta la fecha, la cuestión de la contribución del turismo tanto a la lucha contra la pobreza como a la conservación de la naturaleza genera debate y desacuerdos en el ámbito académico. La complejidad de la realidad, la reciente aparición del turismo en el medio rural, así como las transformaciones rápidas del campo, no han permitido comprender del todo los efectos del TRC en las comunidades rurales. Algunos de los principales temas que se abordan actualmente en las investigaciones académicas son: la evaluación de la sustentabilidad del TRC (Camacho-Ruiz, Carrillo-Reyes, Rioja-Paradela y Espinoza-Medinilla, 2016; Merkel y Kieffer, 2015); los efectos sobre el desarrollo comunitario de esta actividad (Daltabuit, Cisneros, Vázquez y Santillán, 2000; Velázquez-Sánchez, Gómez-Velázquez, Bohórquez Canseco, Solana Vásquez y Pérez Delgadillo, 2015; Zizumbo Villareal, 2007); los cambios socioculturales y económicos que genera (Carrier y Macleod 2005; García-Frapolli, Toledo y Martinez-Alier, 2008; Rios Beltrán, 2016); los factores de éxito y fracaso de las empresas comunitarias de turismo de naturaleza; las discrepancias entre los discursos oficiales de instituciones gubernamentales e internacionales y las realidades observadas (Kieffer, 2011; Simpson, 2008); el papel de los actores locales en la construcción y gestión de las iniciativas comunitarias (Gasca Zamora, López Pardo, Palomino Villavicencio y Martín Mathus, 2010); y las políticas públicas vinculadas a la implementación del turismo alternativo (López Pardo, y Palomino Villavicencio, 2008).

Las discusiones de los académicos dedicados al tema giran en torno al vínculo entre el turismo y las sociedades campesinas, en un contexto de polarización en las posturas sobre los efectos del turismo en las comunidades rurales (Gascón y Milano, 2017; Kieffer y Jouault, 2017). Por un lado, existen estudios que presentan el turismo como una solución milagrosa y una panacea para resolver los problemas del mundo rural (Ashley, Roe y Goodwin, 2001), argumentando que el turismo permite el enriquecimiento de las sociedades locales porque, aunque la actividad sea estrictamente exógena, algunos actores locales siempre están en el centro del proceso de desarrollo turístico. Por el otro lado, otra tendencia afirma que muy pocas veces el turismo cumple sus promesas y que genera impactos negativos en las comunidades donde se implementa (Daltabuit, Cisneros, Vázquez y Santillán, 
2000; Machuca, 2008; Vargas del Río y Brenner, 2013), argumentando que esas políticas turísticas en el medio rural para reducir las desigualdades y luchar contra la pobreza no alcanzan sus metas en la realidad y contribuyen incluso, en algunos casos, a incrementar las desigualdades y a producir nuevas tensiones a nivel local. Esta situación contrasta con el discurso proveniente de las instituciones gubernamentales e internacionales que impulsan dichas iniciativas, predicando una reducción de la pobreza casi automática a través del turismo (омт, 2004). Este desacuerdo profundo plantea preguntarse sobre qué bases se fundamentan esas posturas, a nivel teórico, ideológico y metodológico. ¿Se trata de una interpretación divergente de una misma situación en la que las posturas ideológicas y/o disciplinares predominarían? o ¿es una focalización particular sobre casos de estudio que conciernen iniciativas con resultados diferentes?.

Así, el objetivo de la presente investigación fue analizar el estado del TRC en México, sus alcances y sus relaciones con las comunidades indígenas que lo han implementado desde principios de la década de los noventa. Dichos alcances y relaciones se entienden en un sentido amplio, abarcando así una gran diversidad de temas vinculados con los ámbitos sociales, culturales, económicos, políticos y ambientales del turismo, lo que evidencia el carácter multidisciplinario del trabajo. Finalmente, a partir de los conocimientos existentes sobre estos temas, el presente artículo planteó nuevas pistas de reflexión y necesidades de investigación sobre el turismo rural comunitario.

\section{Metodología}

El presente trabajo pretende sintetizar tendencias de investigación, mediante el cruce de aportes interdisciplinarios, experiencia de campo y reflexión teórica, para situarse en la discusión contemporánea en torno a nuevas perspectivas sobre el desarrollo comunitario. Se trató de generar una estrategia interpretativa para jerarquizar temas de interés y direcciones para futuras investigaciones.

La metodología constó de un análisis crítico de información y de datos obtenidos a través de la implementación de distintas técnicas cualitativas de investigación llevadas a cabo entre 2006 y 2018, tanto en campo como en gabinete. Asimismo, se involucraron estudiantes de diferentes niveles educativos en dichas salidas a campo en las que realizaron prácticas escolares e investigación para sus tesis de grado y/o servicios sociales. Finalmente, el trabajo se nutrió y retroalimentó de numerosos encuentros, pláticas y ponencias con otros académicos afines con el tema en espacios académicos como congresos y seminarios, tanto nacionales como internacionales. En el caso del presente trabajo, no hay entonces un instrumento diseñado y aplicado como tal para recolectar datos, sino que fueron varios instrumentos en distintos momentos y con distintos estudios de caso, implementados por el autor y/o otros académicos, cuyos resultados y hallazgos han sido retomados y organizados para discutirlos en el presente artículo.

Las técnicas constaron de una revisión bibliográfica sobre temas de turismo en el medio rural, en publicaciones nacionales e internacionales, abarcando una gran variedad de 
temas para reflejar con la mayor exactitud posible la diversidad, no sólo de enfoques sobre el TRC, sino también de temas analizados por la comunidad científica. En cuanto al trabajo en campo, se aprovechó como fuente de información una experiencia de 12 años en el marco de distintos proyectos de investigación, aglunos de ellos conducidos en el marco de consultoría para diversas oNG con unos 40 centros ecoturísticos del país. En estos trabajos de campo, se aplicaron técnicas de investigación cualitativa, tales como observación, observación participante, entrevistas semiabiertas y a profundidad, charlas informales y talleres participativos.

Posterior a estos resultados y reflexiones sobre los aportes del TRC al desarrollo comunitario, se planteó una discusión basada en la identificación de tendencias consensuadas por la comunidad académica sobre los temas vinculados con el TRC, lo que permitió detectar temas de investigación pendientes y posibles nichos de trabajos posteriores. Es importante agregar que esta etapa necesitó de un ejercicio de generalización, con todos los límites que aquello plantea, para poder distinguir cuáles son los acuerdos a los que la mayoría de los investigadores han llegado, y cuáles son todavía los puntos de divergencias. Por otra parte, el trabajo intentó dar un panorama general de los temas abordados sobre el TRC y su vínculo con el desarrollo comunitario, por lo que constó de un abordaje multidisciplinario. El TRC, al igual que cualquier forma de turismo, se vincula con varios ámbitos de la sociedad. Por lo tanto, las investigaciones sobre la relación entre el turismo y las comunidades rurales en México son muy diversas y necesitan de un acercamiento interdisciplinario para intentar abarcar la diversidad de temas y enfoques que se manejan en los estudios.

\section{El turismo rural comunitario en México}

Para sistematizar los alcances del TRC de manera holística, se decidió abordar la relación del TRC con la sociedad, de manera amplia; retomando sus principales ámbitos: sociopolítico, económico, ambiental y cultural.

\subsection{Financiamiento y políticas públicas: los inicios caóticos de la inversión pública en el TRC}

Partiendo de la premisa de que, a escala nacional e internacional, financiamientos importantes han sido movilizados para desarrollar el TRC y que los resultados en la realidad han sido muy fluctuantes, un primer punto de encuentro entre las investigaciones relacionadas con el tema ha sido las deficiencias en la implementación del TRC en su inicio, etapa para la que se reconoce la poca coherencia de los diferentes órganos de financiamiento (López Pardo, 2012). Diversos estudios han realizado un censo de aproximadamente 17 instituciones, a través de 57 programas, de los cuales 24 con evidencia de apoyos económicos, participaron en la inversión pública para el desarrollo del TRC entre los años 2006 y 2012 (López Pardo y Palomino Villavicencio, 2014). En particular, el Programa Turismo Alternativo en Zonas Indígenas (PTAZI), conducido del 2007 al 2012 por la Comisión Nacional para el 
Desarrollo de los Pueblos Indígenas (CDI), hoy Instituto Nacional de los Pueblos Indígenas (INPI), ha sido uno de los principales programas de apoyo a la implementación del TRC, reportando 999 organizaciones apoyadas, con una inversión pública de 983.41 mil millones de pesos entre el 2008 y el 2012 (Consejo Nacional de Evaluación de la Política de Desarrollo Social, 2013).

Sin embargo, la coordinación entre estas dependencias y programas resultó muy complicada, el resultado fue una inversión poco coherente y complementaria. El rumbo del desarrollo del turismo en el medio rural no ha sido claramente identificado en un inicio y ha propiciado una situación confusa basada en la idea de que el turismo era una solución milagrosa, sin tener en realidad objetivos y procedimientos bien definidos. Particularmente, la inversión casi exclusiva en infraestructura y casi nula en capacitación, con diseños preestablecidos y poca participación local, provocó mucha desilusión en las comunidades que se habían formado elevadas expectativas. El enfoque de las políticas públicas asistencialistas en TRC, al haberse enfocado solamente en infraestructura hotelera, no ha permitido a las comunidades apropiarse de esta nueva actividad económica, ni de adquirir las capacidades requeridas por ella, dando como resultado el "azote de la cabañitis"; es decir, la existencia de una infraestructura de hospedaje de cabañas, bastante similares unas con las otras, ubicadas en comunidades indígenas, pero vacías de todo contenido. Conviene también mencionar que la presencia del turismo fue acompañada por la creación de nuevas infraestructuras en las comunidades rurales tales como carreteras, redes telefónicas, centros de salud, entre otras, que, aunque el turismo no haya sido la única razón para ello, ha contribuido a la creación y mejoramiento de infraestructura.

La falta de capacitación y de promoción al inicio de los proyectos comunitarios, identificada por varios actores e investigadores, obstaculizó y, en el mejor de los casos, hizo más lento el despegue de los centros ecoturísticos. La transición de una actividad productiva generalmente agrícola, forestal y/o artesanal, hacia una actividad terciaria nueva como lo es el turismo presentó retos que muchas veces los actores tuvieron que resolver sobre la marcha; la propia experiencia, más que los conocimientos sobre el turismo, permitió despejar el camino.

Por otra parte, la cohesión social y la organización colectiva fueron elementos claves que sostuvieron los procesos, de manera que varios proyectos han logrado consolidarse, aunque el camino fue lento y tedioso para los actores que los sostienen. También con el paso del tiempo el tema de la capacitación se volvió central para que los proyectos pudieran despegar, y principalmente la Secretaría de Turismo (Sectur), la Comisión Nacional para el Desarrollo de los Pueblos Indígenas (CDI) a través del PTAzI, y el Fideicomisos Instituidos en Relación con la Agricultura (FIRA) a través del Programa de Financiamiento para el Turismo Rural, empezaron a acompañar a las comunidades en su proceso de manejo de actividades turísticas. Lo anterior permitió consolidar las primeras iniciativas implementadas en los años noventa e intentar no repetir los mismos errores con las nuevas iniciativas. En particular, se hicieron esfuerzos notables en los ámbitos de la capacitación, comercialización y promoción de los servicios. 
Finalmente, el análisis de la relación entre el turismo y las sociedades rurales a nivel político-institucional no sería completo si no se mencionara que, en regiones específicas, el turismo ha sido usado como una propuesta de control político y territorial del Estado, justo a través de la creación de nueva infraestructura. Casos han sido documentados y analizados bajo este marco de análisis como por ejemplo en la Reserva de Montes Azules en el Estado de Chiapas (Martinelli, 2004).

\subsection{Actividades turísticas del TRC}

Otro elemento analizado por la comunidad científica es la importancia de los recursos locales, tanto naturales como culturales. Una observación común ha sido que la oferta turística de los centros ecoturísticos, por lo menos al principio de su desarrollo, no era clara ni bien pensada. El modelo de TRC, en lugar de enfocarse en los recursos locales se ha repetido de un lado al otro, presumiendo sus contribuciones infalibles al desarrollo y la conservación del medioambiente. Por consecuencia, no se ha pensado ni construido una oferta turística basada en las especificidades locales de cada comunidad, en donde la convivencia con los habitantes y las actividades propuestas estén en el centro de la actividad. Esta situación está evolucionando, y cada vez son más las reflexiones de los actores locales en cuanto a vincular las prácticas tradicionales (agrícolas, artesanales, culturales, etc.) con actividades turísticas. Hoy en día, los centros ecoturísticos ofrecen talleres artesanales, visitas a parcelas, participación en actividades agrícolas como parte de sus servicios. Los únicos casos en donde la diversificación de la oferta turística en el medio rural no se ha dado, es en el caso de los lugares con un flagship (Sharpley, 2007), es decir un atractivo turístico muy llamativo, tal una cascada o un volcán, en donde finalmente las comunidades solamente ofrecen este atractivo. El caso de Las Nubes en Chiapas con su cascada, o de Angahuan con el volcán Paricutín, donde no se ofrece ninguna otra actividad que estos recursos naturales, son un ejemplo de esta situación. En contraste, las comunidades que no atraen a los turistas por sí solas deben construir una oferta turística diversificada y basada en sus propios recursos locales. Ahora bien, si bien es cierto que el TRC permite revalorizar prácticas productivas tradicionales, también se han documentado casos en donde la cultura, a través de la reproducción de ritos o ceremonias, queda sujetada a simples fines turísticos (Santana, 2009).

\subsection{Turismo rural comunitario y conservación}

El desarrollo del turismo en el medio rural, y en particular en zonas indígenas, coincidió, tanto en periodos como en lugares, con la implementación de políticas públicas orientadas a la conservación de los recursos naturales y a su uso y aprovechamiento sustentable. Así, es común que la creación de Áreas Naturales Protegidas (ANP) o que la implementación de programas de conservación, coincida con la implementación de iniciativas turísticas en el medio rural. En algunos casos el programa de conservación tiene que ver con la protección de alguna especie (por ejemplo, el centro ecoturísticos Ara Macao Las Guacamayas, 
con el programa de protección de Las Guacamayas); en otros casos tiene que ver con la protección de un ecosistema (los manglares, los bosques, el desierto, la selva, etc.), pero la coincidencia temporal y geográfica entre turismo y conservación es bastante común. Por otra parte, las iniciativas de TRC tienden a ofrecer una infraestructura de bajo impacto ambiental, integrada al paisaje y que promueve materiales ecológicos. Ahora bien, algo que hasta la fecha no queda demostrado, es el aporte del turismo a la conservación. La implementación del turismo en zonas rurales siempre se ha presentado, ademas de una propuesta económica para el campo, como una actividad que permitiría disminuir la presión sobre el ecosistema, transitando de actividades productivas extractivas a una actividadturística- que necesite de un ecosistema protegido. Algunas investigaciones revelan que el ecoturismo puede aportar de manera indirecta a la conservación, al evitar actividades destructivas y extractivistas;fomentar actividades a favor del medioambiente; capacitando a los actores, sensibilizando a los turistas, entre otras (Obombo Magio y Velarde Valadez, 2018). Sin embargo, se han documentado casos tales como La Ventanilla, Oax. (Vargas del Río y Brenner, 2013) o en la Península de Yucatán (García-Frapolli, Ramos-Fernández, Galicia y Serrano, 2009), en los que el ecoturismo y los programas institucionales que lo acompañan, la llegada de nuevos actores, las disputas internas de poder y los conflictos por el uso de la biodiversidad hacen más vulnerable la ecología local, afectando la cohesión social y la confianza mutua existente, lo que genera más conflictos y debilita las formas tradicionales de tomas de decisiones. Entonces, si bien la justificación de los beneficios del ecoturismo para la conservación parece muy clara en términos de políticas públicas, a la hora de analizar si realmente el turismo ha contribuido a la conservación de los recursos naturales, resulta que las investigaciones no son tan contundentes. En todo caso, concuerdan en que el ecoturismo, como actividad económica, por sí solo no es suficiente para evidenciar beneficios directos hacia la conservación.

\subsection{Especialización vs diversificación económica}

También se ha analizado cómo la conservación de la biodiversidad, la agricultura tradicional y el ecoturismo pueden presentarse como actividades económicas complementarias y estrategias de desarrollo interesantes para las comunidades rurales (García-Frapolli et al., 2007; García-Frapolli, Toledo y Martinez-Alier, 2008). La implementación del TRC se ha pensado desde sus inicios como un complemento económico para las comunidades rurales, al participar en un sistema productivo diversificado que contribuya así a la economía familiar campesina (Cañada, 2015). Se han documentado casos en los que las empresas ecoturísticas comunitarias y privadas pueden proporcionar a los pobladores una actividad económica rentable, que los arraigue a la comunidad y disminuya la necesidad de emigrar por motivos económicos (Piñar Álvarez, Nava Tabladai y Viñas Olivaii, 2011).

Si bien todavía no existe una tendencia clara a nivel nacional de reconversión o de transición de actividades productivas hacia el sector de servicio como el turismo, en ciertos espacios, como en la Península de Yucatán (estados de Quintana Roo, Yucatán y Campeche), la presión del turismo es tal que existen trabajos que han documentado una tendencia 
hacia la especialización turística y la pérdida de actividades tradicionales, tales como la apicultura, la agricultura tradicional o bien la producción de carbón (Ríos Beltrán, 2016). Y aunque el turismo no es el único responsable de la pérdida de actividades tradicionales, el equilibrio entre la diversificación productiva en el sector rural y la especialización hacia una actividad económica es de difícil alcance, y aun cuando al inicio del desarrollo del turismo otras actividades subsisten y conviven, la tendencia hacia la especialización es cada vez más fuerte. El mantenimiento de un sistema económico diversificado en el medio rural pasa por una toma de conciencia importante de los actores locales que permita entender las ventajas de tal diversificación sobre un sistema especializado, ya que la diversificación reduce los riesgos de depender de una sola actividad económica.

\subsection{Factores de éxito y fracaso}

Una de las principales líneas de investigación relacionada con el TRC -aún en construcción-, es el análisis de los factores de éxito y fracaso de las empresas comunitarias de turismo (López Pardo, 2012; Kieffer, 2018). Una cuestión sin resolver tiene que ver con las circunstancias en las que las comunidades rurales reciben y se apropian de algún proyecto turístico y con los procesos que ocurren en la construcción de iniciativas de TRC. Autores como Sofield (2003) plantearon que los beneficios de una iniciativa de TRC residen más en el proceso de su construcción -y todo lo que conlleva a nivel de organización comunitaria y de procesos internos- que en los resultados mismos, económicos por ejemplo. Si bien es cierto que a través de investigaciones participativas en las que se ha reconocido la percepción subjetiva de los actores locales, aquellos han identificado la cohesión social de la comunidad y la organización colectiva como factores claves en el éxito de sus proyectos ecoturísticos (Kieffer, 2018), la trayectoria de las experiencias de TRC en México desde mediados de los noventa nos ha mostrado sin embargo que varias comunidades que incursionaron en el tema turístico no han logrado despegar, no necesariamente por falta de organización, sino por ingresos económicos demasiado bajos como para animar a la gente a seguir. Así, es común en las relatorías de las comunidades escuchar que al inicio del proyecto el número de participantes en la comunidad era mucho más elevado, pero varios de sus compañeros fueron renunciado en el camino. Así, además de los beneficios intangibles que el propio proceso de organización colectiva genera, es imprescindible la existencia de beneficios económicos para que la actividad perdure. En este aspecto, se ha de reconocer que hasta la fecha son pocas las iniciativas que son realmente autosuficientes y que han logrado convertirse en proyectos económicos consolidados. La mayoría de los proyectos de TRC siguen dependiendo de apoyos gubernamentales para subsistir, los ingresos generados cubren apenas los gastos. El crecimiento lento de la actividad, el trabajo voluntario por cargos, una remuneración baja y proyectos que desaparecen cuestionan cada vez más el mito del turismo como solución de los problemas del medio rural.

Otro análisis realizado acerca de los factores de éxito y de fracaso, tiene que ver con las experiencias previas de organización colectiva que han tenido las comunidades rurales antes de emprender una actividad turística. Este aspecto es a menudo mencionado por los 
actores locales, precisando que antes del turismo se organizaron socialmente para otra actividad, sea aquella productiva para la constitución de cooperativas agrícolas, de conservación ambiental, de orden religiosa o bien de defensa del territorio entre otras. Pienso, por citar algunos ejemplos, en los Pueblos Mancomunados en la Sierra Norte de Oaxaca para la gestión y defensa de su territorio (Palomino Villavicencio, Gasca Zamora y López Pardo, 2016), en el movimiento cooperativo indígena de la Sierra Nororiental de Puebla, la Tosepan Kali, así como en la gestión comunitaria de las cooperativas de la comunidad de Nuevo San Juan Parangaricutiro, en Michoacán. La experiencia acumulada en estas iniciativas cuenta indudablemente para otros proyectos colectivos y facilita o dificulta formas de organización social colectiva para el turismo. Este análisis es prácticamente común a todos los centros ecoturísticos en el país y constituye un punto de partida fundamental en el estudio del turismo rural comunitario.

Relacionado con lo anterior, y vinculado con el papel de las comunidades locales en la construcción de las iniciativas de TRC, las relaciones que los responsables locales tejen con otros tipos de actores, sean estos gubernamentales, privados o de otras comunidades involucradas en procesos similares, han sido identificadas como un elemento de suma importancia en el proceso de los proyectos comunitarios de turismo (López Guevara, 2014).

\section{Discusión y nuevas pistas de investigación}

\subsection{Capacitación, criterios de calidad, participación y formas de gestión}

Una vez que la comunidad decide emprender el desarrollo turístico, uno de los temas centrales para su continuidad es el de la profesionalización. Destaca el papel de la capacitación en el desempeño de los proyectos de TRC, que en muchos casos ha sido acompañada de inversiones públicas y sancionada por certificaciones oficiales. Llaman la atención los esfuerzos desmesurados de las comunidades por entrar en los procesos de certificación tanto gubernamentales como internacionales. Los centros ecoturísticos exhiben con mucho orgullo sus reconocimientos y diplomas de certificación, como si tenerlos fuera sinónimo de éxito y de buen servicio. La tecnicidad de los expedientes para certificarse obliga en muchos casos a las comunidades a contratar asesores externos, a veces con financiamiento propios y en otros casos con apoyos gubernamentales, pero esta tendencia hacia todo tipo de certificación esconde probablemente otras reflexiones necesarias sobre qué tipo de servicio se quiere dar, qué actividades realizar, cómo vincular a la comunidad con la actividad turística, etc. La intención aquí no es despreciar el papel de las certificaciones sino cuestionar su carácter cuasi obligatorio para no ser excluido de las redes institucionales en TRC, como si el no estar certificado fuera sinónimo de mala calidad y estarlo significaría buena calidad.

Otro tema para analizar es el papel de los jóvenes en la actividad turística en las comunidades rurales. Tal como se ha observado, los socios y encargados de los proyectos turísticos son generalmente gente mayor, y se nota poca presencia de los jóvenes en el fortalecimiento de la actividad. Existen, sin embrago, casos en donde jóvenes de la comunidad se 
capacitan en turismo para después poner a disposición sus conocimientos en el proyecto de su comunidad de origen. Estos casos se han documentado en Yucatán y Quintana Roo, por ejemplo; es decir, en lugares donde los flujos turísticos permiten ingresos económicos que motivan a los jóvenes a involucrarse y regresar a su comunidad. Pero la realidad es más contrastante y, en la mayoría de los casos, los jóvenes que estudian turismo, aunque vengan de alguna comunidad en donde sí hay una actividad turística, pocas veces regresan porque los salarios y perspectivas laborales son muy bajas, por lo que prefieren insertarse en actividades turísticas más convencionales.

Otro tema de análisis poco abordado son las formas de gestión de las actividades de TRC. Si bien encontramos en la mayoría formas de gestión colectiva, existen diferentes tipos, cada una presenta características particulares y remite a formas organizativas distintas. Así, no es lo mismo una gestión colectiva de un proyecto turístico mediante una cooperativa que mediante la Asamblea de Bienes Comunales o de los ejidatarios, o bien de un grupo de personas de la comunidad. El análisis de estas particularidades y de los resultados que han producido nos podría dar algunos elementos de reflexión y aportar argumentos a la discusión mencionada anteriormente acerca de los factores de éxito y de fracaso de los proyectos de turismo rural comunitario.

\subsection{Conservación, protección ambiental, sustentabilidad}

La contribución como tal del turismo a la conservación es un asunto no resuelto que merece investigaciones para aportar elementos de discusión al famoso paradigma del turismo (o ecoturismo) como propuesta para la conservación. Para ello, no cabe duda de la necesidad de realizar estudios inter y transdisciplinarios que combinen enfoques cuantitativos y cualitativos, tanto de las ciencias sociales como de las ciencias naturales. La complejidad a la que llegó la relación sociedad-naturaleza nos obliga a abordar la realidad desde nuevos enfoques para superar los límites del abordaje disciplinario, que, aunque sigue teniendo relevancia en casos particulares, no permite entender de manera holística los problemas vinculados con el turismo y la sustentabilidad. De igual manera, para sacar conclusiones de la capacidad del turismo alternativo para fomentar el desarrollo comunitario sustentable, se necesitan herramientas conceptuales adaptadas y protocolos para aplicarlas. La ecología política, las ciencias de la sostenibilidad, las epistemologías del sur, la economía social y solidaria, las teorías feministas, son conjuntos de corrientes que nos permiten salir del encajonamiento de las disciplinas tradicionales para enfrentar los retos actuales planteados por la sociedad y particularmente por la actividad turística. Estas nuevas corrientes plantean el diseño de herramientas específicas para analizar la relación hombre-naturaleza en el prisma del turismo. Indicadores de sustentabilidad (Camacho Ruiz, Carrillo Reyes, Rioja Paradela y Espinoza Medinilla, 2016; Merkel y Kieffer, 2015), herramientas participativas, historias de vida, entre otras, pueden ser técnicas valiosas para reorientar la investigación en turismo y así llegar a niveles mayores de complejidad. Se necesita llevar a cabo una reflexión sobre las definiciones y los criterios de la sustentabilidad para poder diagnosticar el alcance de los logros en la materia. 


\subsection{Interpretaciones divergentes de los impactos del TRC : cómo incluir las perspectivas subjetivas}

Un aspecto pendiente en los estudios sobre turismo es la percepción misma de los actores locales sobre el resultado de la iniciativa de TRC, desde su propia perspectiva y no desde la perspectiva gubernamental o de la academia. Tal vez encontraríamos que una iniciativa resulta ser un éxito desde el punto de vista de los actores y un fracaso para el programa gubernamental en el cual se insertó la iniciativa, o viceversa, y ello nos obligaría a replantear los factores de éxito de las iniciativas de TRC. Su principal factor de éxito podría residir, por ejemplo, en la capacidad de los actores de generar formas de gobernanza participativa en sus comunidades. En este contexto, el análisis de los procesos que ocurrieron en la implementación de actividades de TRC, y de las interpretaciones locales de dichos procesos, son elementos esenciales para entender las repercusiones actuales tanto a nivel económico como social, cultural y ambiental en el ámbito del desarrollo comunitario. El renovado interés por el concepto de individuo como actor y modificador del espacio por sus prácticas tanto económicas como sociales y culturales, es una de las tendencias observadas por la geografía del turismo desde los años 2000 en un afán de renuevo cultural (Hiernaux, 2008), planteando la necesidad de construir una mirada menos estructural y más atenta al hombre. Basado en la experiencia subjetiva de los actores, el estudio de los fenómenos sociales desde la manera en cómo los sujetos experimentan e interpretan el mundo social constituye un reto importante en la investigación social, y en particular en el ámbito turístico.

Ello abre la discusión sobre la necesidad de reconstruir las prácticas de los actores involucrados en una iniciativa de TRC, desde su propia perspectiva, y cuestionar la naturaleza del éxito de dichas iniciativas. Se necesita para ello que se realicen trabajos de sistematización participativa de las experiencias actuales de TRC, con la finalidad de rescatar el proceso conducido por las comunidades y confrontar su propio acercamiento a esa actividad turística específica con el planteado por los académicos, las ong y gobiernos. Ello permitiría, por ejemplo, determinar patrones de éxito o de fracaso de iniciativas de TRc bajo la propia perspectiva de los actores locales, lo que permitiría dar un paso grande para la orientación de políticas públicas futuras.

Del otro lado de la cadena turística, se destaca el papel del TRC en la sensibilización de los turistas y en los efectos que estas iniciativas puedan tener sobre los turistas en términos sociales, humanos y ambientales. El TRC, presentado como un espacio de sensibilización entre culturas distintas y cuestiones ambientales, permitiría en teoría participar en la construcción de una sociedad más empática, más cercana a la naturaleza y consciente de las injusticias sociales. Pero esta situación todavía no ha quedado demostrada, y serían bienvenidos trabajos desde la psicología y la sociología para entender si existe esa relación benéfica entre el TRC y la construcción de un mejor futuro. 


\subsection{Hacia nuevas definiciones del desarrollo comunitario}

Los análisis realizados sobre la relación entre el TRC y las sociedades campesinas, y particularmente sobre el papel de las comunidades indígenas en la construcción y gestión del turismo a nivel local, invitan a desplazar el objetivo inicial tradicional del emprendimiento del TRC -la diversificación de la economía, una alternativa de desarrollo, la protección de los recursos naturales, la diversificación de la oferta turística- hacia uno centrado en las teorías de empoderamiento de los actores locales y de la expansión de las libertades y emancipación de los individuos (Sofield, 2003), del desarrollo comunitario y del modo de vida de las comunidades rurales, enfatizando la importancia del proceso de construcción de esas iniciativas. Tal como se mencionó, el marco institucional, el contexto socioeconómico y la temporalidad histórica en la que han aparecido las iniciativas de TRC en México, han sido comunes a prácticamente todas las comunidades rurales. Sin embargo, si bien se empiezan a deslindar patrones comunes en la construcción y en la gestión de las iniciativas, los resultados, varían de un lugar a otro, de una comunidad a otra. Esto quiere decir que es preponderante la manera en la que las comunidades se apropian de los proyectos, tejen redes con otros actores y se desempeñan finalmente en este sector del turismo. En el estudio de esta interfaz entre políticas públicas impuestas desde arriba y las respuestas de las comunidades rurales a dichas políticas, reside toda la complejidad del análisis de la relación entre turismo y sociedades rurales.

El concepto de capital social comunitario, entendido como un conjunto de interacciones que abarcan los conceptos de confianza, normas de reciprocidad, redes de participación civil, reglas y leyes que permiten conformar relaciones complejas de cooperación y gestión en un grupo, más allá de las redes sobres las cuales se apoya el capital social individual (Durston, 2000), aplicado al turismo, ha permitido entender y analizar fenómenos en torno a los factores de éxito y de fracaso del TRC (Barbini, 2008; López Guevara, 2014). La suma de las experiencias, vivencias y aprendizajes realizados durante el proceso de implementación de la iniciativa de TRC puede ser considerada como una forma de capital social y humano que, aunque tarde en dar frutos porque al principio no se nota, a la hora de mantener un proyecto a largo plazo resulta provechoso. En este sentido, la participación de los actores locales en la construcción de las iniciativas de TRC (Tosun, 2000) juega un papel esencial en el éxito de su gestión años después. Las comunidades que no han logrado apropiarse de los proyectos, que no han podido tejer relaciones con otros actores y que han mantenido una actitud pasiva de sencillas receptoras de política pública, tienen más dificultades que aquellas que tuvieron un proceso participativo más avanzado (Kieffer, 2018).

La hipótesis que se plantea para abrir la discusión en torno a la investigación sobre el TRC consiste en pensar que el proceso que siguen las comunidades rurales hacia el emprendimiento del TRC importaría más que el resultado mismo de la actividad. El TRC merece ser analizado más como un proceso de construcción de nuevas habilidades cuya finalidad es crear cohesión comunitaria a la vez que potenciar las libertades individuales. Así como se necesita desplazar el objetivo de las iniciativas del TRC, se necesita adaptar el objeto de estudio de las investigaciones académicas sobre este. Quizás éste sea el giro necesario 
en la investigación, ubicar el TRC dentro de un proceso de expansión de libertades y dentro de un gran marco de desarrollo comunitario, en el sentido amplio y holístico de la palabra; es decir, desde temas de autonomía política y administrativa, de manejo de recursos naturales, de formas de organización social, etc., que permita a las comunidades rurales construir modos de vida que respondan a sus necesidades actuales, y no solo como una actividad económica que genere nuevos ingresos. En este sentido, la noción de desarrollo debe ser entendida no solo como un crecimiento económico, sino como un proceso de cambio social construido por los actores locales y que toma en cuenta las características culturales, sociales, organizacionales y ambientales de cada territorio (Vachon, 2001).

El estudio de la interacción de los procesos sociales, culturales, económicos y ambientales que ocurren en torno al TRC se presenta como un tema pendiente de investigación, tanto para producir conocimientos académicos y entender qué implicaciones conlleva el turismo, como para atender en el futuro problemas vinculados al turismo y al desarrollo comunitario. Dentro de los estudios académicos pendientes sobre el TRC, la sistematización participativa de experiencias (Bickel, 2005), cuya finalidad sería la de comprender las prácticas para mejorarlas, es un reto muy importante.

\subsection{Vincular los estudios locales y las dinámicas globales}

Para cerrar la discusión, cabe reconsiderar la oposición de discursos mencionada al inicio de este trabajo. ¿De donde viene esa oposición tan marcada? ¿Estos dos discursos se basan en estudios de casos distintos, en los cuales en algunos se podrían observar beneficios del turismo alternativo en las comunidades locales y en otros efectos negativos? 0 bien, ¿esas posturas interpretativas opuestas podrían cada una "verificar" su "teoría" en un mismo estudio de caso?

Desde luego, el carácter muy polémico del turismo, aún más abordado como herramienta de desarrollo en el medio rural, explica en parte esa polarización e incitaría a responder que no hay casos exitosos ni fracasos, sino que son las posturas interpretativas de cada investigador las que determinarían el efecto positivo o negativo del turismo en las comunidades rurales. Se plantea entonces la hipótesis de que los análisis sobre la relación del turismo con las sociedades locales están sesgados por cuestiones disciplinarias y enfoques conceptuales, así como en función de la procedencia (académica, social, privada, gubernamental) y los marcos teóricos de quienes conducen los estudios. Según esta hipótesis, para un mismo estudio de caso, estaríamos frente a una situación de producción de conocimientos "corroborados" de teorías explicativas locales opuestas, contradictorias, en las cuales al mismo tiempo un análisis afirmaría, por ejemplo, la reducción de la pobreza, y otro observaría la creación de nuevas desigualdades, impactos negativos, etc. (Kieffer, 2015). Sin embargo, asumir que son las posturas teóricas que orientan las interpretaciones y los análisis sobre el turismo alternativo conllevaría a invisibilizar el papel de los actores locales en la conformación de las iniciativas turísticas y sería caer en un determinismo teórico que ha quedado obsoleto tras los renuevos de las ciencias sociales y particularmente de la geografía cultural. 
Así, resulta necesario conducir investigaciones que permitan salir de esa oposición de discursos. Para ello, se hace hincapié en la posibilidad de abordar estudios a través de un enfoque microsocial; es decir, que "parte de lo específico, de la realidad social y del turismo hacía la comprensión, producción de conceptos o construcción de conocimiento" (González Damián y Palafox, 2014, p. 816). Stone y Stone (2011) observaron que existen relativamente pocas evaluaciones sobre los impactos de las políticas públicas del TRC a nivel local, como mecanismo de lucha contra la pobreza y de conservación de los recursos naturales y aun menos emergieron desde las propias experiencias y percepciones locales. Finalmente, resulta más interesante centrarse en las trayectorias de los actores locales, las condiciones y cambios territoriales después de la llegada del turismo, las relaciones sociales creadas a través del encuentro en el TRc, etc. Este enfoque abre nuevas perspectivas de análisis para salir de la oposición de discursos mencionada, que polariza y sesga el debate en torno a la relación entre turismo y desarrollo. Partir de la realidad social, de los estudios de caso para conceptualizar el TRC, permite no reproducir corrientes de pensamientos fijos y evitar imponer o hacer corresponder obligatoriamente la teoría y los conceptos sobre la realidad.

Sin embargo, este acercamiento desde la realidad hacía la conceptualización de la relación turismo-desarrollo no es suficiente, pues es necesario salir del análisis aislado del estudio de caso, y agregar a este enfoque microsocial un acercamiento comparativo al fenómeno del TRC. Esta tendencia nos lleva a conducir investigaciones a escalas micro, comunitarias, con el riesgo de tener una multitud de estudios de caso muy interesantes, pero poco conectados entre sí. Así, la perspectiva regional de trabajos como el de García de Fuentes, Jouault y Romero (2015) permite entender dinámicas regionales vinculadas con el TRC y se presenta como un enfoque necesario para llegar a enmarcar las dinámicas turísticas locales en procesos regionales. El análisis de este juego de escalas parece muy evidente, pero a la fecha poco se ha trabajado, aunque permitiría entender y relacionar procesos locales que parecieran desvinculados pero que en realidad responden a patrones institucionales, económicos, políticos, ambientales y culturales similares en algunos aspectos. Este vaivén entre lo local y lo regional necesita del manejo de enfoques y herramientas sociales y geográficas especializadas, por lo cual la geografía podría realizar aportes interesantes.

Así, el reto de la investigación en TRC quedaría en hacer dialogar estudios de casos muy detallados para así reconstruir las prácticas y las dinámicas tanto a escalas locales como regionales. El riesgo entre llegar a niveles de profundización importantes sin ver más allá de su estudio de caso y quedarse en niveles de generalización sin profundizar en aspectos locales siempre existirá en el ámbito del análisis de las relaciones entre el turismo alternativo y las sociedades rurales, pero nos toca a nosotros, como académicos, resolver esta disyuntiva y hacer dialogar enfoques teóricos y diferentes acercamientos a la investigación. 


\section{Conclusiones}

Este trabajo se enfocó en analizar la relación entre el turismo rural comunitario (TRC) y el desarrollo comunitario en México, sus retos y alcances, para entender mejor el fenómeno y sus implicaciones para las comunidades rurales, a nivel social, cultural, económico y ambiental. Obviamente las tendencias presentadas no son exhaustivas y el fuerte dinamismo que observamos en el desarrollo del TRC en México también está presente en los temas de investigación. Sin embargo, existen en la literatura algunos temas recurrentes entre los investigadores que reflejan observaciones en el terreno, así como los cambios ocurridos en relación con la actividad turística de las comunidades que decidieron implementarla.

El fuerte vínculo existente entre las políticas internacionales de desarrollo y de conservación, las políticas públicas en los mismos ámbitos y la implementación de iniciativas comunitarias de turismo ha sido documentado en numerosas ocasiones. De allí, se ha abordado el TRC como resultado de políticas sociales y de conservación, situación que influyó de manera directa en su desarrollo y en la predominancia de una visión oportunista del turismo por distintos actores. En muchos casos, los esquemas de implementación del TRC han sido reproducidos de manera arbitraria de una comunidad a la otra, sin tomar en cuenta las condiciones específicas de cada lugar, y con niveles muy bajos de apropiación de la iniciativa por parte de los actores locales. Varios estudios coinciden en que la inversión pública en infraestructura no ha permitido que las comunidades rurales pudieran desarrollar capacidades reales para manejar iniciativas de TRC, haciendo hincapié en la necesidad de capacitación, promoción y comercialización de este tipo de turismo. También se ha analizado el papel del turismo en los cambios productivos de las comunidades rurales, cómo ha incidido en varios casos en la transición de una economía diversificada a una especializada, lo que ha contribuido a crear una situación de precariedad al depender exclusivamente de la actividad turística.

Impactos, factores de éxito y fracaso han sido analizados por varios investigadores, argumentando que la organización colectiva de las comunidades constituye uno de los principales pilares para el desarrollo de esta actividad. La elaboración de metodologías participativas responde al análisis convergente de muchos investigadores que ubican el punto neurálgico del TRC en la apropiación de las iniciativas por los actores involucrados. Por otra parte, existen líneas de investigación para el estudio del TRC que podrían ser desarrolladas en el futuro, que tienen que ver con la evaluación de los criterios de sustentabilidad, tanto desde una perspectiva subjetiva como objetiva. Asimismo, se ha manifestado en distintos espacios académicos la necesidad de llevar a cabo investigaciones con enfoque regional y geohistórico, para así salir del estudio de caso aislado y empezar a teorizar a partir de la práctica. Para ello, resulta importante sistematizar las experiencias, analizar el contexto de las tomas de decisiones para evaluar las iniciativas de TRC, con la finalidad de esbozar patrones de implementación y así entender mejor las dinámicas turísticas a escala regional. 


\section{Agradecimientos}

Investigación realizada gracias al Programa UNAM-DGAPA-PAPIIT IA30401 Inventario Nacional del Turismo Alternativo en México.

\section{Fuentes citadas}

Ashley, C., Roe, D. y Goodwin, H. (2001). Pro-poor tourism strategies: Making tourism work for the poor. Pro-Poor Tourism Report Núm. 1. Overseas Development Institute, Londres, Nottingham: Russell Press.

Ávila Romero, A. (2015). Análisis del turismo alternativo en comunidades indígenas de Chiapas, México. Études Caribéennes, 31-32. Dol: 10.4000/etudescaribeennes.7601.

Barbini, B. (2008). Capacidades locales de desarrollo a través del turismo: reflexiones para su abordaje. En César D., A. y Arnaiz B., S.M. (Eds.), Turismo y desarrollo: crecimiento y pobreza (pp. 207-227). México: Universidad de Guadalajara.

Bickel, A. (2005). La sistematización participativa para descubrir los sentidos y aprender de nuestras experiencias. El Salvador: Red Alforja / Funprocoop.

Camacho-Ruiz, E., Carrillo-Reyes, A. Rioja-Paradela, T. M. y Espinoza-Medinilla, E. E. (2016). Indicadores de sostenibilidad para el ecoturismo en México: estado actual. Revista LiminaR. Estudios Sociales y Humanísticos, 16(1), 156-168.

Cañada, E. (2015). La comercialización del turismo comunitario en América Latina. Anuario de Estudios Centroamericanos, 41, 159-189.

Cañada, E., y Gascón, J. (2007). Turismo y desarrollo: Herramientas para una mirada crítica. España: Fundación Luciérnaga-Acción por un Turismo Responsable.

Carrier, J. y Macleod, D. (2005). Bursting the bubble: The sociocultural context of ecotourism. The Journal of the Royal Anthropological Institute, 11, 315-334.

Consejo Nacional de Evaluación de la Política de Desarrollo Social (Coneval) (2013). Informe de la Evaluación Específica de Desempeño 2012-2013, Valoración de la información de desempeño presentada por el Programa Turismo Alternativo en Zonas Indígenas (PTAZI) de la Comisión Nacional para el Desarrollo de los Pueblos Indígenas. México: Autor, 9 p.

Daltabuit, M., H. Cisneros, L. M. Vázquez y E. Santillán (2000). Ecoturismo y desarrollo sustentable: impacto en comunidades rurales de la Selva Maya. Cuernavaca, México: Universidad Nacional Autónoma de México, Centro Regional de Investigación Multidisciplinaria.

Dehoorne, O., Tatar, C. y Theng, S. (Dir.). (2013). Tourisme et lutte contre la pauvreté: approche théorique et études de cas. Études Caribéennes, vol. 24-25.

Durston, J. (2000). ¿Qué es el capital social comunitario? Santiago de Chile: Naciones Unidas, Comisión Económica para América Latina y el Caribe (Cepal) / División de Desarrollo Social. (Serie Políticas Sociales Núm. 38. )

García de Fuentes, A, Jouault, S. y Romero, D. (2015). Atlas de turismo alternativo de la península de Yucatán. México: Cinvestav-Unidad Mérida. 
García-Frapolli, E. et al. (2007). Biodiversity conservation, traditional agriculture and ecotourism: Land cover/land use change projections for a natural protected area in the northeastern Yucatan Peninsula, Mexico. Landscape and Urban Planning, 83.

García-Frapolli, E., Ramos-Fernández, G., Galicia, E. y Serrano, A. (2009). The complex reality of biodiversity conservation through Natural Protected Area policy: Three cases from the Yucatan Peninsula, Mexico. Land Use Policy, 26, 715-722.

García-Frapolli, E., Toledo, V. y Martinez-Alier, J. (2008). Adaptations of a Yucatec Maya multiple-use ecological management strategy to ecotourism. Ecology and Society 13(2): 31.

Gasca Zamora, J., López Pardo, G., Palomino Villavicencio, B. y Martín Mathus, A. (2010). La gestión comunitaria de recursos naturales y ecoturísticos en la Sierra Norte de Oaxaca. México: unam, Instituto de Investigaciones Económicas / Academia Mexicana de Investigación Turística.

Gascón, J. y Milano, C. (2017). El turismo en el mundo rural ¿Ruina o consolidación de las sociedades campesinas e indígenas? Pasos, Revista de Turismo y Patrimonio Cultural.

Girard, A. y Schéou, B. (2012). Le tourisme communautaire à l'épreuve des illusions culturaliste et participative, l'exemple d'une expérience au Bénin. Tourisme, Territoires et Développement, 157, 67-80.

González-Damián, A. y Palafox, A. (2014). Sociología del turismo en español. Revisión exploratoria de artículos publicados en revistas iberoamericanas 2003-2013. Estudios y Perspectivas en Turismo, 23.

Hiernaux, D. (2008). El giro cultural y las nuevas interpretaciones geográficas del turismo. GEOUSP-Espaço e Tempo 23: 177-187.

Kieffer, M. (2015). La relación turismo-comunidades rurales: salir de la oposición de discursos. En M. Nel-lo Andreu, B. Campos Cámara, A.P. Sosa Ferreira, Temas pendientes y nuevas oportunidades en turismo y cooperación al desarrollo (pp. 106-114). México: Red Internacional de Investigadores en Turismo, Cooperación y Desarrollo.

Kieffer, M. (2018). Turismo Rural Comunitario y organización colectiva: un enfoque comparativo en México. PASOS Revista de Turismo y Patrimonio cultural, Vol. 16, 2. Dol: 10.25145/j.pasos.2018.16.030

Kieffer, M. y Jouault, S. (2017). Nouveaux enjeux de la recherche en tourisme au Mexique: l'analyse des relations tourisme et développement rural. En C. Guibert et B. Taunay (Dirs.), Le tourisme au prisme des sciences sociales. Ancrages et postures épistémologiques (pp. 50-68). Francia: Presses Universitaires de Rennes.

López Guevara, V. M. (2014). El capital social en las empresas indígenas de turismo. Su análisis y dinamización en la red Expediciones Sierra Norte, México (Tesis de doctorado) Universitat de Girona: España:.

López Pardo, G. y Palomino Villavicencio, B. (2008). Políticas públicas y ecoturismo en comunidades indígenas de México. Teoría y Praxis, 5, 33-50.

López Pardo, G. (2012). Políticas gubernamentales para el desarrollo del turismo naturaleza en comunidades y pueblos indígenas en México. Patrimonio Cultural y Turismo, 19.

López Pardo, G. y Palomino Villavicencio, B. (24 de septiembre 2014). Las paradojas del financiamiento gubernamental al turismo de naturaleza en México. (Ponencia) Congreso de Investigación Turística Aplicada: Zapopan, Jalisco. 
Machuca, J. A. (2008): Estrategias turísticas y segregación socioterritorial en regiones indígenas. En A., Castellanos G., y J. A., Machuca, Turismo, identidades y exclusión. México: Universidad Autónoma Metropolitana / Juan Pablos Editores.

Manyara, G., y Jones, E. (2007). Community-based tourism enterprises development in Kenya: An exploration of their potential as avenues of poverty reduction. Journal of Sustainable Tourism, 15, 628-644.

Martinelli, L. (2004). Nuevos desalojos en los Montes Azules: La Reserva de la Biosfera, el Corredor Biológico Mesoamericano y el papel de Conservación Internacional. CIEPAC, 393.

Merkel, N. y Kieffer, M. (2015). Diagnóstico de sustentabilidad turística: evaluación participativa y generación de propuestas para una iniciativa de turismo rural comunitario (TRC). En A. Gámez, A. Palafox y M. Gutiérrez. La construcción del espacio turístico: procesos, actores e impactos. Memorias del IX Congreso Internacional de la Academia Mexicana de Investigación Turística (pp. 260-275). México: Instituto Sudcaliforniano de Cultura-Academia Mexicana de Investigación Turística-Universidad Autónoma de Baja California Sur.

Obombo Magio, K. y Velarde Valadez, M. (2018). Ecoturismo y conservación: Perspectivas y prácticas en las reservas de la biósfera de Los Tuxtlas, México y Maasai Mara, Kenia. Dimensiones Turísticas, 2(2), 53-77.

Organización Mundial del Turismo (2004). Turismoyatenuación de la pobreza. Recomendación para la acción. Madrid: World Tourism Organization. DoI: $10.18111 / 9789284407019$.

Palomino Villavicencio, B., Gasca Zamora, J. y López Pardo, G. (2016). El turismo comunitario en la Sierra Norte de Oaxaca: perspectiva desde las instituciones y la gobernanza en territorios indígenas. El Periplo Sustentable, 30, 6-37.

Piñar Álvarez, A., Nava Tabladai, M. E., Viñas Olivaii, D.K. (2011). Migración y ecoturismo en la Reserva de la Biosfera de Los Tuxtlas (México). PASOS. Revista de Turismo y Patrimonio Cultural, 9(2), 383-396. Dol: 10.25145/j.pasos.2011.09.033.

Ríos Beltrán, F. I. (2016). Análisis de los medios de vida sostenibles en el área de protección de flora y fauna otoch ma 'ax yetel kooh (Tesis de licenciatura) Escuela Nacional de Estudios Superiores Unidad Morelia, unam: Michoacán.

Santana B., L. (2009). Eculturismo en territorio Wixarika (Tesis de maestría) Facultad de Filosofía y Letras, unAm: México.

Sharpley, R. (2007). Flagship Attractions and Sustainable Rural Tourism Development: The Case of the Alnwick Garden, England. Journal of Sustainable Tourism, 15, 125-143.

Simpson, M. C. (2008). Community benefit tourism initiatives: A conceptual oxymoron? Tourism Management, 29, 1-18.

Sofield, T. H. B. (2003). Empowerment for sustainable tourism development. Amsterdam: Pergamon.

Stone, L. S. y Stone, T. M. (2011). Community-based tourism enterprises: challenges and prospects for community participation; Khama Rhino Sanctuary Trust, Botswana. Journal of Sustainable Tourism, 19, 97-114.

Tosun, C. (2000). Limits to community participation in the tourism development process in developing countries. Tourism Management, 21, 613-633.

Vachon, B. (2001). El desarrollo local. Teoría y práctica. Reintroducir lo humano en la lógica del desarrollo. España: Ediciones Trea. 
Velarde Valdez, M., Maldonado Alcudia, A. V., Gómez Nieves, S. (Coords.) (2015). Referentes teóricos del turismo alternativo. Enfoque en comunidades rurales. México: Universidad de Guadalajara / Universidad de Occidente / UCLA Program on Mexico / Profmex/World / Juan Pablos Editor.

Vargas del Río, D. y Brenner, L. (2013). Ecoturismo comunitario y conservación ambiental: la experiencia de La Ventanilla, Oaxaca, México. Estudios Sociales, 21(41), 32-63.

Velázquez-Sánchez, R. M., Gómez-Velázquez, J., Bohórquez Canseco, M. G., Solana Vásquez, O. M. y Pérez Delgadillo, A. (2015). Ecoturismo y desarrollo local de comunidades indígenas en México. Revista Global de Negocios, 3(3), 67-76.

Zizumbo Villarreal, L. (2007). El turismo en comunidades rurales: práctica social y estrategia económica (Tesis de doctorado) Facultad de Filosofía y Letras, unam: México. 